\title{
Der "Deutsche Gruß" in Briefen. Zur historischen Soziolinguistik und Pragmatik eines verordneten Sprachgebrauchs
}

\author{
Klaas-Hinrich Ehlers (Frankfurt/Oder)
}

\begin{abstract}
The official decree, in 1933, to use "Heil Hitler" and "Mit deutschem Gruß [With German greetings]" as greetings, constituted a massive intervention in the everyday use of language in the German population. The study examines in what time frame and in what domains of written communication the new forms of greeting were established. Sources include guidelines and sample letters from Nazi era style guides as well as a body of authentic business letters from the 1920s to 1940s. The development described in the study is characterized as a phase of forcibly accelerated modernization in the long-term history of European communication routines. The introduction of the Hitler salute is identified as a premature and passing episode in the multidimensional transition from feudal courtesy of the vertical distance towards a bourgeois/contemporary courtesy of the horizontal distance.
\end{abstract}

\section{$1 \quad$ Verordnete Grußformen}

Am 13. Juli 1933 ordnete Reichsinnenminister Wilhelm Frick per Runderlass an alle Reichsbehörden und Landesregierungen "für den dienstlichen Bereich die Einführung des deutschen Grußes an" (Overesch et al. 2001: 376). Der Deutsche Gruß, der vor 1933 im Umkreis der NSDAP gebraucht wurde, war schon bald nach der Machtübernahme, am 19. April 1933, für Polizei und Wehrmacht vorgeschrieben worden. Im Dezember 1933 ging Frick über die bisherigen Anweisungen noch einmal hinaus und verordnete die Anwendung des Hitlergrußes auch für die Schule:

Der Lehrer tritt zu Beginn jeder Unterrichtsstunde vor die stehende Klasse, grüßt als erster durch Erheben des rechten Armes und die Worte "Heil Hitler"; die Klasse erwidert den Gruß durch Erheben des rechten Armes und die Worte "Heil Hitler". (Overesch et al. 2001: 8.284)

Auf gleiche Weise war die Schulstunde zu beenden, bei Begegnungen von Lehrer und Schüler "innerhalb und außerhalb der Schule" genügte "das Erheben des rechten Arms in angemessener Haltung" (ebd.). Innerhalb weniger Monate war der Geltungsbereich des Hitlergrußes von der Exekutive über die Staatsbediensteten allgemein auf das Schulwesen erweitert worden und somit zur verbindlichen Grußform weiter Teile der deutschen Bevölkerung in wichtigen Bereichen ihres Alltags geworden.

Grüße etablieren in der Regel kommunikative Kontakte und stiften somit wenigstens für die Dauer dieses Kontaktes soziale Gemeinschaften. ${ }^{1}$ In der spezifischen verbalen und nonverbalen Ausprägung ihres Grußverhaltens geben sich Gesprächspartner zugleich zu verstehen, wie sie die grüßend eingegangene Sozialität fundiert und strukturiert wissen wollen.

\footnotetext{
1 Dass der Gruß mit seiner Kontaktfunktion "zur Bestätigung der Zusammengehörigkeit" der Gesprächspartner beitragen bzw. bei einer Erstbegegnung ein soziales "Band stiften" und beim Abschiedsgruß dieses "Band für die Zukunft bestätigen" kann, stellt schon der Klassiker der modernen Grußforschung Eibl-Eibesfeldt (1971: 196) heraus.
} 
Die Wahl eines Grußmusters ist also als Index situativer und sozialer Wirklichkeitskonstruktion bzw. -affirmationen lesbar. (Scharloth/Okamura 2010: 50)

So konnte (und sollte) im historisch besonderen Fall des Hitlergrußes die Grußgemeinschaft als eine Gesinnungsgemeinschaft markiert werden. Die gewählte verbale oder gestische Grußform wurde hier zum Zeichen geteilter nationaler oder nationalsozialistischer Gesinnung. Mitunter löste sich der Deutsche Gruß ganz aus dem Kontext von Kontakteröffnung oder beendigung und wurde zum rituellen Bekenntnis der Zugehörigkeit zu dieser Gesinnungsgemeinschaft. Victor Klemperers Tagebuch hält aus der Zeit unmittelbar nach Machtübernahme den folgenden Bericht einer Bekannten fest.

Sie erzählt, wie fanatisiert die Schwestern und Wärter ihres Krankenhauses sind. Sie sitzen um den Lautsprecher. Wenn das Horst-Wessel-Lied gesungen wird (jeden Abend und auch sonst), stehen sie auf und erheben den Arm zum NS-Gruß.2

So wie das Ausbleiben eines erwarteten Grußes oder Gegengrußes im Alltag in der Regel ein empfindliches Symptom für eine kommunikative Störung oder einen sozialen Beziehungsabbruch gewertet wird, so bekam die Verweigerung des Hitlergrußes zusätzlich die Bedeutung einer demonstrativen politischen Aussage. Im totalitären Staat konnte das Ausscheren aus der Gesinnungsgemeinschaft drastische Sanktionen nach sich ziehen. Klemperer erwähnt in seinem Tagebuch im Sommer 1933 den Fall einer Frau, deren Ehemann "Kraftfahrer bei der Reichspost" war:

Sie erzählt mit Tränen in den Augen: ein Kollege ihres Mannes Knall auf Fall entlassen, weil er nicht mit Armaufheben gegrüßt hat. ${ }^{3}$

Klemperer selbst geriet durch den Zwang zum Hitlergruß an der Hochschule in Dresden immer wieder in quälende Gewissensnot und Existenzängste:

Aber mir wird von diesem Händeaufheben buchstäblich übel, und daß ich mich immer wieder daran vorbeidrücke, wird mir noch einmal den Hals brechen. ${ }^{4}$

Die Einführung des neuen Sprach- und Gestengebrauchs war also beim Hitlergruß von Beginn an mit existentieller Bedrohung jeder Abweichung verbunden. Wir haben es hier mit einem äußerst durchgreifenden Fall politischer Sprachlenkung zu tun. Wie reagierte nun die deutsche Sprechergemeinschaft auf diesen Eingriff in die bisherigen alltagssprachlichen Konventionen?

In den Tagebüchern Victor Klemperers, der als "rassisch" Verfolgter jede Veränderung des Sprachgebrauchs mit alarmierter Aufmerksamkeit verzeichnete, lesen wir beispielsweise am 28. Juli 1933, also 15 Tage nach dem entsprechenden Runderlass des Reichsinnenministers, die folgende Beschreibung der Verhältnisse an der Technischen Hochschule Dresden:

Die letzten Semestertage brachten der TH noch den Geßlerhut: Zwang zum "Hitlergruß". [...]

Bisher grüßten mich kleine Beamte und Kollegen mit Kopfnicken wie sonst, und ich erwiderte ebenso. Auf Kanzleien aber sah ich die Angestellten immerfort die Hand heben. Und Fräulein Mey erzählte uns, dass man es strikte durchführe. (Klemperer 1995: 44)

Nahezu ein Jahr später berichtet ein Bekannter Klemperers von einer Reise nach Süddeutschland:

Ich war eben in Süddeutschland. Da hört man sehr selten das "Heil Hitler" - meist "Grüß Gott". 5

\footnotetext{
2 Klemperer (1995: 21). Eintrag vom 10. April 1933.

${ }^{3}$ Klemperer (1995: 46). Eintrag vom 10. August 1933. Bauer (1988: 117ff.) zeigt an einer Reihe von Beispielen, dass die Verweigerung des Hitler-Grußes durch "Entlassung, Gefängnis, selbst KZ-Haft" (ebd.: 123) und im äußersten Falle auch durch Ermordung sanktioniert wurde. Vgl. Allert (2005: 89 f.).

${ }^{4}$ Klemperer (1995: 87). Eintrag vom 15. Februar 1934. Vgl. z.B. den Eintrag vom 2. März 1934 ebd. S. $93-94$.
} 
Diese beiden kurzen Notizen deuten schon darauf hin, dass die Durchsetzung des Deutschen Grußes ganz offensichtlich nicht so umfassend und durchgreifend erfolgte wie von politischer Seite wohl erwünscht. Es ist vielmehr mit zeitlichen und auch regionalen Unterschieden zu rechnen. Der Gebrauch des Deutschen Grußes dürfte auch zwischen verschiedenen sozialen Gruppierungen (z.B. Beamte, Arbeiter) und kommunikativen Domänen deutlich geschwankt haben.

Die Frage nach Verlauf und Reichweite der erzwungenen Änderung des deutschen Grußverhaltens steht, wie jede Frage nach der Geschichte des Sprachgebrauchs, ${ }^{6}$ vor einem ernsten Quellenproblem. Gewiss birgt schon ein einzelnes Werk wie das Tagebuch Victor Klemperers eine Fülle von Beobachtungen zum Deutschen Gruß. Ähnliche Quellen könnten herangezogen und auch Zeitzeugen könnten heute noch befragt werden. Solange es sich aber um den mündlichen Sprachgebrauch handelt, wird es immer schwierig bleiben, den genauen Kontext der berichteten oder erinnerten Grußphänomene zu rekonstruieren. Für den schriftlichen Sprachgebrauch ist dagegen eine große Fülle von primären Quellen überliefert. Schriftliche Grüße sind in archivierten Briefen in ihren authentischen Ko-text eingebettet, derartige Quellen geben außerdem meist sehr dichte Hinweise auf den ursprünglichen kommunikativen $\mathrm{Zu}-$ sammenhang des jeweiligen Textdokuments. Ich möchte mich daher in dieser historiographischen Studie zum Deutschen Gruß in erster Linie auf das schriftliche Grußverhalten konzentrieren. Bevor ich hierzu eine Sammlung authentischer Briefe aus der Zeit des Nationalsozialismus als Belegkorpus auswerte, möchte ich aber Briefsteller und Benimmbücher dieser Zeit zu Rate ziehen. Derartige Sprachratgeber können zwar nicht eigentlich als Primärquellen gelten, weil sie den damaligen Sprachgebrauch stets laienlinguistisch reflektieren, bewerten und vorschreiben. Die präskriptive Zielsetzung der zeitgenössische Ratgeberliteratur basiert aber häufig auf einem erstaunlich breit ausgebauten deskriptiven Fundament. Überdies enthalten Briefsteller oft ausdifferenzierte Sammlungen von Musterbriefen und Textproben, die den Anspruch erheben, realitätsnahe gestaltet und in den Sprachalltag der Leser übertragbar zu sein. Auf diese Weise bilden Briefsteller die kommunikative Realität ihrer Zeit - mit gewissen Brechungen - ab, andererseits wirken ihre auflagenstark verbreiteten Vorgaben ihrerseits auf die Sprachwirklichkeit zurück.?

\section{Der 'Deutsche Gruß' in der zeitgenössischen Ratgeberliteratur und in ihren Musterbriefen}

Was also sagen die Sprachratgeber der NS-Zeit zur Verwendung des Deutschen Grußes in der schriftlichen Kommunikation? Die expliziten Handlungsanweisungen und Gebrauchs-

\footnotetext{
5 Klemperer, (1995: 114), Eintrag vom 13. Juni 1934. Auch Bauer (1988: 121) stellt fest, dass die Durchsetzung des (mündlichen) Hitlergrußes in den Regionen, in denen traditionell mit "Grüß Gott" gegrüßt wurde, nicht so weit ging wie in den "Guten Tag"-Regionen Deutschlands. Vgl. Allert (2005: 86), der von einem "Nord-SüdGefälle" der Zustimmung zu der neuen Grußkonvention spricht.

6 Während Untersuchungen zur Sprache im Nationalsozialismus bisher vorwiegend den Wortschatz und die Idiomatik oder aber die politische Rhetorik bzw. den Argumentationsstil untersucht haben, möchte ich in meinem Betrag einen, freilich sehr engen, Ausschnitt aus dem alltäglichen Sprachgebrauch im Dritten Reich rekonstruieren. Allerts Buchessay zum Deutschen Gruß konzentriert sich vor allem auf die gestischen Aspekte des Hitler-Grußes und stützt sich nur auf disparate Einzelquellen oder anekdotische Erinnerungsberichte. Sein phänomenologischer Ansatz bekommt die situative, soziale und diachronische Differenzierung des damaligen Grußverhaltens kaum in den Blick, Allert (2005).

7 Aus diesem Grund stützte sich schon Ermert bei seiner Untersuchung zu den Briefsorten auf die Briefstellerliteratur: "Auch Briefsteller-Autoren [arbeiten] nicht ohne Bezug zur realen Briefkommunikation. Andernfalls würden ihre Erzeugnisse wohl keine Käufer finden; [...]. Abgesehen von gewissen Verzerrungen der Briefrealität (in Richtung "Schönheit") können Briefe aus Briefstellern also durchaus als mit realen Briefen kompatibel angesehen werden" (Ermert 1979: 16). Die starke Wirkungsmacht der Briefsteller lässt sich beispielsweise deutlich an vielen authentischen "Bittschriften, Briefen und Berichten" "kleiner Leute" aus dem 19. Jahrhundert beobachten, denen Siegfried Grosse et al. einen materialreichen Sammelband gewidmet haben (Grosse et al. Hrsg. 1989).
} 
beschreibungen sind hier häufig sehr umfassend. Im dreiteiligen Ratgeber Briefe, die Eindruck machen, beschreibt einer der drei Verfasser die Verhältnisse 1936 wie folgt:

In Deutschland ist jetzt ganz allgemein und ausnahmslos bei allen Gelegenheiten, geschäftlichen wie privaten Briefen, der Schluß des Briefes gebräuchlich "Mit Deutschem Gruß" oder "Heil Hitler!" (Freymann 1936: 27)

Hier wird also zunächst ein gleichsam universeller Gebrauch der beiden Varianten des Deutschen Grußes im gesamten Briefverkehr behauptet. Es folgt dann aber eine Einschränkung, die mit dem ausnahmslosen Gültigkeitsanspruch dieser Behauptung auffällig in Kontrast steht:

Selbstverständlich kann man in rein persönlichen Briefen auch jede andere Schlussformel anwenden. (ebd.)

Tatsächlich findet sich dann in der etwa einhundert Seiten umfassenden Sammlung von Musterbriefen, die im Abschnitt "Privatbriefe" dieses Briefstellers abgedruckt wird, nur ein einziger Beleg des Deutschen Grußes. Ein Entschuldigungsschreiben von Eltern an die Lehrerin ihrer Tochter schließt hier "Mit deutschem Gruß!" (ebd.: 106). Offensichtlich klafft zwischen dem allgemeinen ideologischen Anspruch an den Sprachgebrauch und den Briefmustern, die der Verfasser im Bereich der privaten schriftlichen Kommunikation für realitätsnahe hält, ein bemerkenswerter Abstand.

Anspruch und mutmaßliche Textwirklichkeit kommen aber auch im Abschnitt "Geschäftsbriefe und Bewerbungsbriefe" desselben Briefstellers nicht vollständig zur Deckung. "In Briefen an Behörden und sehr große Unternehmen", so heißt es hier, würde "heutzutage vielfach überhaupt keine Schlußformel mehr angewendet" (Lehmann 1936: 152). Im übrigen wird behauptet:

In Deutschland schließt man auch in Schreiben an Behörden usw. mit dem Gruß "Heil Hitler!" oder "Mit deutschem Gruß!" (ebd.).

Sofern die Schlussformeln in den Beispielbriefen zur Geschäftskorrespondenz überhaupt ausgeführt werden, finden sich neben Briefschlüssen mit Deutschem Gruß aber auch Belege für andere Schlussformeln. Und den Gruß "Heil Hitler" verwendet der Autor des Abschnitts zum Geschäftsbrief in seinen Musterbriefen gar nicht. Für "geschäftliche Rundschreiben", "Glückwunschbriefe", "geschäftliche Entschuldigungsbriefe", "Werbebriefe" und "Bewerbungsbriefe" bringt er Textmuster sowohl "mit deutschem Gruß" als auch mit Variationen "ergebener Grüße" oder "bester Empfehlungen" am Schluss. Die Wahl zwischen diesen Grußformen ist dabei recht deutlich auf den jeweiligen Adressaten des Musterbriefes abgestimmt: Glückwunschbriefe zu Jubiläen von Vereinen schließen beispielsweise "mit deutschem Gruß", Glückwunschschreiben zu Amtsjubiläen, Geschäftsübernahmen oder Geburtstagen von Einzelpersonen enden dagegen mit "ergebenen Grüßen". Der Werbebrief eines Handwerkers an einen Stadtrat schließt entsprechend "mit deutschem Gruß", ein fiktiver Schneidermeister bedenkt seinen Privatkunden in seinem Werbeangebot "mit ergebensten Grüßen".

Die "Schreiben an Behörden, Eingaben und Urkunden", die der dritte Teil des Briefstellers aufführt, kommen zum größten Teil ohne jede Schlussformel aus (Mancke 1936). Für den "Verkehr mit Verwaltungsbehörden" bringt der Autor neben Texten ohne Schlussformel ausschließlich Beispielbriefe mit "Heil Hitler" (an den Bürgermeister, an das Finanzamt) oder "mit deutschem Gruß" (an den Regierungspräsidenten, an den Polizeipräsidenten). Für Briefe an Dienststellen der NSDAP wird der Gruß "Heil Hitler!" empfohlen (ebd.: 328).

Eine ähnliche, wenn auch keineswegs völlig übereinstimmende Ausdifferenzierung der Gebrauchsdomänen des Deutschen Grußes nimmt der Briefsteller von Curt Elwenspoek vor, der ebenfalls aus dem Jahr 1936 stammt. Wie im vorher vorgestellten Briefsteller fasst Elwenspo- 
ek die Gebrauchskontexte des Deutschen Grußes in den Einleitungspassagen seines Buches zunächst denkbar weit:

Jeder Deutsche setzt heute unter seinen Brief: "Mit Deutschem Gruß" oder "Heil Hitler". Dieser Gruß gilt jedem ohne Rang- und Standesunterschied. Das ist der Gruß der deutschen Volksgemeinschaft, der du und ich und in der Regel auch der Empfänger angehören. (Elwenspoek 1936:

Für die behördliche und die geschäftliche Korrespondenz entsprechen die Musterbriefe bei Elwenspoek ganz dieser allgemeinen Vorgabe. Beschwerden bei der Polizeidirektion, Eingaben beim Finanzamt oder Terminfestsetzungen bei Gericht werden ebenso durchgängig mit "Heil Hitler" oder dem "deutschen Gruß" unterzeichnet wie die abgedruckten Muster für geschäftliche Briefsorten wie Bewerbung, Bestellung, Mahnung oder Beschwerde.

Für den Bereich des privaten Briefwechsels schränkt aber auch Elwenspoek die universelle Gebräuchlichkeit des Deutschen Grußes ausdrücklich ein:

Man soll auch den Namen seines Führers und Reichskanzlers nicht unnützlich führen, und niemand muß fürchten, für einen schlechten Nationalsozialisten zu gelten, wenn er in solchen ganz privaten Schreiben dem Gedenken an Führer und Vaterland nicht noch mit Worten eigens Ausdruck verleiht. (ebd. 113) ${ }^{8}$

Elwenspoeks Mustertexte zum Liebesbrief und zum Beileidsschreiben weichen daher durchgängig auf andere Schlussformeln aus. Aus Negativbeispielen für misslungene Beileidsschreiben wird in der vorbildhaften Umformulierung jeweils auch der unangebrachte Deutsche Gruß getilgt (ebd.: 104, 108). Elwenspoek rät "in solchen Briefen von Mensch zu Mensch alles, was an das Geschäftliche erinnern könnte", (ebd.: 108) zu vermeiden. Anders als der Briefsteller Briefe, die Eindruck machen, hält Elwenspoek offensichtlich auch den Glückwunschbrief für nicht vereinbar mit dem Deutschen Gruß, und zwar auch dann nicht, wenn es um eher distanzierte Glückwünsche an einen "sehr verehrten" oder gar "hochverehrten" "Herrn Kommerzienrat" geht (ebd.: 99-102).

Andererseits schließen Elwenspoeks Beispiele für "ganz private Schreiben" aber gelegentlich mit dem Deutschen Gruß. Eine Einladung zum Abendessen "im kleinsten Kreise" beispielsweise ist mit "Heil Hitler" unterzeichnet (ebd.: 110). Während der Eingeladene in diesem Fall allerdings gesiezt und mit Titel angeschrieben wird, bringt Elwenspoek auch Musterbeispiele für "freundschaftliche Briefe" zwischen Duzfreunden, unter die ein "Heil Hitler" gesetzt wird: Ein mustergültiger Bittbrief an einen Freund schließt beispielsweise "Mit herzlichen Grüßen von Haus zu Haus und Heil Hitler! / Dein alter Friedrich." (ebd. 128). Der Hitlergruß drang demnach mitunter sehr weit in die Sphäre der privaten Korrespondenz vor.

Mit dem Blick auf diese beiden Briefsteller ist die Variationsbreite des schriftlichen Grußverhaltens bereits hinreichend abgegrenzt, in dem sich auch andere Sprachratgeber der Zeit bewegen. Ich fasse meine Beobachtungen an Benimmbüchern und Briefstellern der NS-Zeit und ihren Musterbriefsammlungen zusammen. Diese Zusammenfassung beruht auf der vergleichenden Untersuchung von neun verschiedenen Benimmbüchern und Briefstellern, die in Nazi-Deutschland publiziert wurden. ${ }^{9}$ Das große Korpus der dort abgedruckten Musterbriefe bietet eine gute Grundlage, den variierenden Gebrauch der Grußformeln auch in seinen quantitativen Verhältnissen näherungsweise zu bestimmen: Anders als manche Briefratgeber nach 1933 vollmundig behaupten, ist der Deutsche Gruß in der zeitgenössischen schriftlichen Kommunikation offensichtlich nicht ausnahmslos in allen Situationen gebraucht worden. Die

\footnotetext{
${ }^{8}$ Dieses Zitat ist bemerkenswert nicht nur wegen der zeittypischen religiösen Stilisierung des Politischen, sondern weil - ungewollt - die "Furcht" als ein wesentliches Motiv für die Verwendung des Deutschen Grußes benannt wird.

9 Briefe die Eindruck machen (1936), Elwenspoek (1936), Haluschka (1938), Leder (o.J.), Martin (o.J.), Meister (1934 [?]), Rohr (o.J.), Teichmann (o.J.), Wilda (1939).
} 
"Musterbriefe zur Anregung" (Rohr o.J.: 234) des Lesers für die eigene Korrespondenz, die den Ratgebern meistens beigegeben werden, zeigen vielmehr deutlich, dass der Gebrauch des Deutschen Grußes nach kommunikativen Domänen, aber auch nach dem Bekanntheitsgrad und Statusunterschieden zwischen Absender und Empfänger der Schriftstücke gestaffelt war. Am treffendsten umreißt der Universalbriefsteller von J. Rohr am Ende der 1930er Jahre die tatsächlichen Gebrauchsbedingungen des schriftlichen Hitlergrußes, wenn er resümiert,

dass wir an uns Fernstehende, an Vorgesetzte, an Behörden oder unter Geschäftsbriefe den Gruß schreiben, der heute unter allen Deutschen gilt. (Rohr o.J.: 17)

Die zeitgenössischen Sprachratgeber lassen vor allem in ihren Mustersammlungen erkennen, dass die Verwendung von Schlussformeln mit "Heil Hitler" oder "Mit deutschem Gruß" von der behördlichen Korrespondenz über die Geschäftsbriefe bis zum privaten Schriftwechsel in ihrer Frequenz stark abnahm. In der schriftlichen Kommunikation von und mit Behörden und staatlichen Institutionen war demnach überall dort, wo überhaupt Schlussformeln unter die Schreiben gesetzt wurden, das "Heil Hitler!" der prototypische Abschiedsgruß. In diesen Institutionen war der Deutsche Gruß für die Binnen- wie für die Außenkommunikation per Dienstanweisung eingeführt und offenbar im schriftlichen Bereich weitestgehend durchgesetzt worden. Außenstehende, die sich brieflich an Behörden wandten, hatten deren Sprachgebrauch tunlichst zu übernehmen.

Eine Zwischenposition nahm die geschäftliche Kommunikationssphäre ein. Auch hier überwiegen zahlenmäßig deutlich die Musterbriefe mit dem Deutschen Gruß bzw. mit "Heil Hitler!". Sobald aber die Geschäftskorrespondenz in den privaten Handlungsbereich überging, etwa wenn kaufmännische Werbebriefe oder Glückwunschschreiben an Geschäftsfreunde als persönliche Ansprachen konzipiert waren, machte der Deutsche Gruß anderen Grußformen Platz.

Die schriftliche Kommunikationssphäre, in welcher der Deutsche Gruß die vergleichsweise geringste Rolle spielte, war den Musterbriefen zufolge der private Briefwechsel. Hier sind die Befunde zum Grußverhalten nicht nur von Briefsteller zu Briefsteller, sondern zu Teil auch innerhalb der einzelnen Werke etwas uneinheitlich. Dennoch lassen sich für das Vorkommen des Deutschen Grußes auch in der privaten Korrespondenz einige typische Gebrauchsbedingungen angeben. So scheint eine dominante expressive Textfunktion im Brief den Deutschen Gruß tendenziell auszuschließen. Liebes- und Beileidsbriefe wurden offenbar grundsätzlich nicht mit dem Deutschen Gruß abgeschlossen, Glückwunschschreiben meistens dann nicht, wenn sie einzelnen Empfängern als Person galten. Dass die persönliche und soziale Distanz zwischen den Kommunikationspartnern die Verwendung des Deutschen Grußes beeinflusste, ist daran zu erkennen, dass sein Vorkommen in den Musterbriefen typischerweise mit bestimmten Anredeformen korreliert. Die Anrede des Empfängers mit Titel oder Funktionsbezeichnung scheint einen Deutschen Gruß eher nahe gelegt zu haben, Duzbekanntschaften schlossen ihn eher aus. In den Fällen, in denen der Deutsche Gruß dennoch zwischen Duzfreunden oder innerhalb der Familie verwendet wurde, lassen sich häufig thematische Zusammenhänge ausmachen. Neujahrsbriefe innerhalb des engeren Familienkreises schließen gewöhnlich ohne Deutschen Gruß. Dieser wird aber dann in den Briefschluss gesetzt, wenn im vorhergehenden Text von der politischen Lage die Rede war (z.B. Rohr o.J.: 256-259). Während Geburtstagsglückwünsche an Verwandte in der Regel ohne Deutschen Gruß auskommen, kann ein Glückwunschschreiben zur Einsegnung, das den Eintritt des Empfängers in die gesellschaftliche Welt der Erwachsenen in feierlich gehobenen Stil thematisiert, durchaus mit "Heil Hitler! Deine alte Großmutter" (Rohr o.J.: 263) abgeschlossen werden. ${ }^{10}$

${ }^{10}$ In dem Benimmbuch von Hans Teichmann findet sich ein interessanter Hinweis auf eine besondere Beliebtheit des Deutschen Grußes in der Kommunikation unter Männern: "Der Deutsche Gruß (durch Erheben des rech- 
Es bleibt ein zahlenmäßig freilich sehr geringer Rest von nicht dominant expressiven Musterbriefen zwischen guten Bekannten und sogar Duzfreunden, die mit Deutschem Gruß schließen, obwohl sie keine Gegenstände von allgemeinem gesellschaftlichem Belang thematisieren oder das Bestreben einer Gesinnungskundgabe erkennen lassen. Da ist beispielsweise die Einladung eines jungen Mannes an ein "Liebes Fräulein Gerda" zur gemeinsamen Paddelbootfahrt (Rohr o.J.: 274) oder der tröstende und Hilfe anbietende Brief eines Mannes an seinen schwer erkrankten Duzfreund (Elwenspoek 1936: 124), die beide auf "Heil Hitler!" enden. Derartige Musterbriefe können bzw. sollen wohl als Grenzfälle zeigen, wie weit sich der Gebrauchsbereich des Deutschen Grußes von seiner ursprünglich amtlichen Einführung in den privaten Sprachalltag der deutschen Bevölkerung bereits ausgedehnt hatte.

In welchem Verhältnis standen nun die beiden Formulierungsvarianten des Deutschen Grußes "Heil Hitler" und "Mit deutschen Gruß"? Beide Grußformeln konnten für Gesinnungsbekundungen stehen, die einerseits sehr nahe beieinander lagen, deren feiner Unterschied aber dennoch für Zeitgenossen wichtige Ausdrucksmöglichkeiten eröffnete. Nach Victor Klemperer wurde die Briefunterschrift "Mit deutschem Gruß" "in den ersten Jahren von halbtapferen Leuten als Ersatz für 'Heil Hitler' gewagt" (Klemperer 1957: 127), wenn sie ihrer Distanz zum politischen Regime Ausdruck geben wollten, "ohne doch nachweisbare Opposition zu treiben" (ebd.: 126). Vorgeschrieben war, sofern es um die verbale Grußrealisierung ging, allein die Formel "Heil Hitler". Diese Formulierung war daher offizieller als die Wendung "Mit deutschem Gruß". In der Ratgeberliteratur der Zeit wird der Hitler-Gruß außerdem als die modernere Grußformulierung dargestellt und zum Teil ausdrücklich in ein zeitliches Überbietungsverhältnis zu "Mit deutschem Gruß" gesetzt:

In der verflossenen Verfallszeit unseres Volkes hatte dieser Gruß die Bedeutung der nationalen Gesinnung des Schreibers. Die ihn heute noch anwenden, erklären ihn als den heutigen Gruß der deutschen Nation; es ist dann aber nicht recht einzusehen, warum man diesen dann nicht unmittelbar anwendet, also: Heil Hitler! (Rohr o.J.: 17)

Im Briefsteller von Harald v. Wilda werden Formulierungsalternativen von Bewerbungsbriefen, die für "Geschraubtheit hier und Natürlichkeit dort" (Wilda 1939: 89) stehen sollen, unter anderem durch die gewählte Schlussformel unterschieden. Das "Heil Hitler" passt demnach in die "empfohlene neue Form", die als "frisch" und "natürlich" attribuiert wird. "Mit deutschem Gruß" repräsentiert entsprechend die veraltete "Geschraubtheit" (ebd.: 89-91)11. Ebenso wie J. Rohr lässt Harald von Wilda seine Musterbriefe daher, wo immer ein Deutscher Gruß gewählt wird, in allen Gebrauchsdomänen mit "Heil Hitler" schließen. Andere Ratgeberautoren, wie beispielsweise Curt Elwenspoek, stellen die beiden Varianten des Deutschen Grußes in ihren allgemeinen Erörterungen gleich und wechseln auch in ihren Musterbriefsammlungen zwischen beiden Schlussformeln, ohne dass Motive für die jeweilige Wahl er-

ten Armes!) ist nicht nur der Dienstgruß in allen unseren Betrieben, sondern er eignet sich auch für die Straße, besonders im Verkehr der Männer untereinander, ausgezeichnet" (Teichmann o.J.: 45). Dieser Hinweis steht aber in der mir bekannten Ratgeberliteratur der NS-Zeit allein. Er lässt sich auch an den Musterbriefsammlungen der Briefsteller nicht stichhaltig überprüfen, denn die weit überwiegende Mehrzahl der dort fingierten Korrespondenten ist männlich. Es finden sich also kaum geeignete Vergleichspaare von Briefen, aus denen sich Rückschlüsse auf ein möglicherweise geschlechtsspezifisches Grußverhalten ziehen ließen.

11 An anderer Stelle lässt der Verfasser einen "Lehrling der alten Zeit" ein Bewerbungsschreiben "mit Deutschem Gruß" schließen, das als Negativbeispiel misslungener Gestaltung gegeben wird, Wilda (1939: 95-96). 
kennbar wären. ${ }^{12}$ Es finden sich in der Zeit des Nationalsozialismus aber auch Sprachratgeber, die in ihren Briefbeispielen ausschließlich "mit deutschem Gruß" verwenden. ${ }^{13}$

\section{Der 'Deutsche Gruß' in der Geschäftskorrespondenz mit der Deutschen Forschungsgemeinschaft}

Ich möchte meine Beobachtungen an Sprachratgebern und Musterbriefsammlungen nun überprüfen und ergänzen durch die Untersuchung authentischer Sprachproben aus den 1920er bis 1940er Jahren. Ich konzentriere mich dabei auf Briefe aus dem Bereich der behördlichen und geschäftlichen Kommunikation, also auf die Kommunikationsdomäne, die wohl am ehesten und am durchgreifendsten von der Sprachlenkung betroffen war. Sehr geeignetes Belegmaterial findet sich in den so genannten "Einzelfallakten" der Deutschen Forschungsgemeinschaft, die im Bundesarchiv Koblenz liegen. ${ }^{14}$ Die Deutsche Forschungsgemeinschaft war 1920 unter dem Namen "Notgemeinschaft der deutschen Wissenschaft" gegründet worden und hatte sich bald zu der zentralen Institution der staatlichen Forschungsförderung in Deutschland entwickelt, als die sie auch heute noch bzw. wieder tätig ist. Die Subventionierung betraf damals Druckkostenzuschüsse, Forschungs- oder Reisestipendien oder die Beschaffung von Materialien und Apparaten für die Experimentalforschung. Die archivierten Einzelfallakten dokumentieren in unterschiedlicher Überlieferungsdichte die Vergabe staatlicher Förderung für einzelne Wissenschaftler: im Idealfall von der Antragstellung über die fachliche, wirtschaftliche und politische Begutachtung bis hin zur Abwicklung der finanziellen Seite der Förderung. Diese Abläufe erforderten eine ausgedehnte interne und externe Kommunikation, deren schriftliche Seite breite archivalische Spuren hinterlassen hat. Ein außerordentlich umfangreicher Personenkreis war in diese Korrespondenzen einbezogen, Wissenschaftler (etablierte wie Nachwuchskräfte), Beamte, Politiker, Verleger oder Kaufleute aus anderen Firmen und natürlich Vertreter der Forschungsgemeinschaft. Die Korrespondenzpartner unterschieden sich daher häufig recht deutlich in ihrem gesellschaftlichen Status. Die erhaltenen Briefe gliedern sich funktional in eine breite Palette von Briefsorten aus, vorherrschend sind dabei repräsentative (Berichte, Bescheide) und direktive (Anträge, Bitten) Textfunktionen. Von besonderem Interesse war für mich auch der Briefwechsel mit dem deutschsprachigen Ausland, an dem sich die regionale Dimension der beobachteten Entwicklungen ablesen lässt.

Um einen möglichst weiten Überblick über die Variationsbreite der Schlussformeln zu erlangen, habe ich Belegtexte aus möglichst vielen verschiedenen Einzelfallakten ausgewählt, in denen jeweils verschiedene Antragsteller, Gutachter und oft auch unterschiedliche Vertreter der Forschungsgemeinschaft in Kontakt treten, um über die vielfältigsten Anliegen zu korrespondieren. Mein Belegkorpus umfasst insgesamt 300 Briefe $^{15}$ aus 66 verschiedenen Einzelfallakten. Für jeden Jahrgang zwischen 1933 bis 1939 habe ich 30 bis 40 Briefe ausgewertet, so dass der hier vor allem interessierende Zeitraum recht dicht dokumentiert ist. Die Jahre vor 1933 und die frühen vierziger Jahre berücksichtige ich in umfangreichen Stichproben.

In chronologischer Anordnung vermitteln meine Belegbriefe ein recht deutliches Bild von den Verschiebungen im deutschen Grußverhalten. Vor 1933 stand im Zentrum der typischen

\footnotetext{
12 So etwa in dem von Fritz Mancke verfassten dritten Teil des Ratgebers Briefe, die Eindruck machen (1936: 241-347), der den Schreiben an Behörden gewidmet ist. Für "Schreiben an Parteidienststellen" wird allerdings nur "Heil Hitler" vorgeschrieben, ebd: 328.

13 Beispielsweise im Benimmbuch von Hans Martin (o.J.). Auch Gustav Lehmann lässt die von ihm angeführten Mustergeschäftsbriefe stets auf "Mit deutschem Gruß" schließen, wenn ein Gruß angebracht ist, vgl. Lehmann (1936).

14 Bundesarchiv Koblenz, Bestand R 73. Ich danke den Mitarbeitenden im Bundesarchiv für die Unterstützung meiner Recherchen.

15 Nicht adressierte Textsorten wie Lebensläufe, Aktennotizen, Berichte aus Briefanlagen o. Ä. wurden dabei nicht berücksichtigt, da hier ohnehin keine Schlussformeln zu erwarten waren.
} 
Schlussformel demnach die Versicherung der "Hochachtung" gegenüber dem Adressaten. Diese Hochachtung wurde wahlweise als "ausgezeichnete", "vorzügliche" oder "größte", seltener als "vollendete" oder "besondere" attribuiert. Vor allem bei größeren Statusunterschieden erweiterten die Briefschreiber der späten 1920er und frühen 1930er Jahre ihren Briefschluss gern um den Ausdruck ihrer eigenen Ergebenheit gegenüber dem Empfänger. Durch zusätzliche Steigerung der Attribute konnte so die Standardformel "in vorzüglicher Hochachtung" beispielsweise zu "in vorzüglichster Hochachtung Ihr ergebenster" 16 angehoben werden. Der Aufwand der Respekterweisung konnte natürlich auch durch andere sprachliche Mittel noch vergrößert werden. Das zeigt ein Antrag der Dialektologin Anneliese Bretschneider, den sie im Herbst 1932 an den Präsidenten der Forschungsgemeinschaft richtete und wie folgt schloss: "Mit dem Ausdruck meiner ausgezeichneten Hochachtung / bin ich Ew. Exzellenz ganz ergebene". ${ }^{17}$ Als seltene Ausnahme von dieser variantenreichen Versicherung der Hochachtung finden sich in der schriftlichen Korrespondenz zwischen Institutionen gelegentlich Briefe, die sowohl auf eine persönliche Anrede als auch auf eine Schlussformel verzichten. So etwa, wenn der bayrische "Verband für Flurnamensammlung" sich "An die Notgemeinschaft der deutschen Wissenschaft" wendet und sein Anliegen ohne einleitende Anrede und abschließende Grußformeln vorbringt. ${ }^{18}$

An diesem Sprachgebrauch änderte sich mit der Machtübernahme der Nationalsozialisten und den bald ergehenden Erlassen zum Grußgebrauch zunächst kaum etwas. In meinen 30 Belegbriefen des Jahres 1933 finden sich neben der typischen Hochachtungsformel und seltenen schlusslosen Briefen überhaupt nur drei Schreiben, die mit Deutschem Gruß enden. ${ }^{19}$ Als erster im ganzen Korpus wandte sich im Oktober 1933 ein auslandsdeutscher Philologe an die Deutsche Gesandtschaft in Riga und schloss seine Bitte um Vermittlung mit der hybriden Formel: "Ich verbleibe / mit deutschem Gruß / und Heil Hitler / Ihr aufrichtig ergebener"20. In ähnlich hybrider Formulierung schrieb zum Jahresende 1933 der Verlagsausschuss der Forschungsgemeinschaft an den "Hochgeehrte[n] Herr[n] Geheimrat" Prof. Edward Schröder und grüßte "In grösster Hochachtung / und mit deutschem Gruss"21. Nur ein Schreiben des nationalistischen Bundes Deutscher Osten reduziert die Schlussformel schon im Dezember 1933 auf ein vorschriftsmäßiges knappes "Heil Hitler" und kommt auch ohne einleitende Anrede aus. ${ }^{22}$

Bevor ein solches Grußverhalten sich als Norm etablieren konnte, sollte aber noch einige Zeit verstreichen. Ab 1934 gingen die Vertreter der Forschungsgemeinschaft ebenso wie ihre inlandsdeutschen Korrespondenzpartner zunächst dazu über, ihre Briefe "Mit deutschem Gruß" zu schließen. Gelegentlich wurde "mit deutschem Gruß und Heil Hitler" zu einer Schlussformel kombiniert. In manchen Fällen grüßten die Briefschreiber innerhalb Deutschlands 1934 aber noch mit Varianten "vorzüglicher Hochachtung". Dieser heterogene Gebrauchsstand kommt sehr deutlich auch in den Anweisungen zum Grußgebrauch zum Ausdruck, die der

\footnotetext{
16 Mehrfach belegt in Briefen von Verlegern an Professoren bzw. Geheimräte.

17 Brief vom 12.8.1932 (R73/10468), A. Bretschneider war damals 32 Jahre alt. Zeilenwechsel werden hier und in folgenden Zitaten durch Schrägstriche markiert.

18 Schreiben vom 3.9.1931 und Antrag vom 15.6.1932 (R73/16549).

19 Ilse Meisters Schreibtischberater aus der Frühzeit des Dritten Reiches empfiehlt den Deutschen Gruß noch gar nicht. Ihre Muster z.B. für förmliche Bewerbungsschreiben enden - wie mein Belegmaterial aus der Zeit bis Mitte 1933 - ausnahmslos auf Varianten von "in vorzüglicher Hochachtung". Im Übrigen empfiehlt die Autorin für den distanzierten "Verkehrston" "hochachtungsvoll" als Briefschluss (Meister 1934: 88-98 und 100). Krumrey (1984: 693) datiert das mir ohne Jahresangabe vorliegende Buch auf 1934, Datumsangaben im Text zu Folge ist das Manuskript frühestens im Juli 1933 abgeschlossen worden.

${ }^{20}$ Schreiben Prof. Mutschmanns aus Dorpat an Minister O. Reinebeck, Deutsche Gesandtschaft in Reval, vom 28.10.1933 (R73/16822).

21 Schreiben vom 27.12.1933 (R73/14846).

22 Schreiben "an das Präsidium der Notgemeinschaft der deutschen Wissenschaft" vom 22.12.1933 (R73/10468).
} 
damalige Präsident der Forschungsgemeinschaft im Dezember 1933 seinen Mitarbeitern per Rundschreiben auferlegte:

Ich bitte, künftig allgemein in von mir oder in meinem Auftrage zu unterzeichnenden Schreiben an deutsche Volksgenossen, die mit einer Höflichkeitsformel abgeschlossen werden, die Formel "mit deutschem Gruß" anzuwenden. In besonderen Fällen kann hierzu die Erweiterung treten "und in grösster Hochachtung" oder "und in grösster Hochschätzung" oder Entsprechendes [...]. Eine wohlbegründete andersartige Fassung der Schlussformel in Einzelfällen soll hierdurch nicht ausgeschlossen sein. ${ }^{23}$

Von einem solchen Gebrauchsstand aus verengten sich die Gestaltungen der Briefschlüsse in den Folgejahren allmählich immer mehr auf die Formel "Heil Hitler!". Erst 1937 hatte sich der Hitlergruß endgültig als der zentrale und meist auch alleinige Bestandteil der Schlussformel in meinen Belegbriefen durchgesetzt. Ein Zahlenvergleich soll Anfang und Ende dieser Entwicklung veranschaulichen. Von 25 Briefen reichsdeutscher Absender des Jahres 1934 verwendeten 15 in ihrer Schlussformel "Mit deutschem Gruß", in 8 Fällen war "Mit deutschem Gruß" dabei alleinige Grußformel. "Heil Hitler" wurde in Kombinationen zehnmal am Briefschluss verwendet, trat aber überhaupt nur einmal isoliert auf. Neben drei Briefen ohne jede Schlussformel endeten 1934 auch noch zwei Briefe mit der traditionellen Versicherung der Hochachtung, ohne diese mit Varianten des Deutschen Grußes zu kombinieren. Im Jahr 1937 hatten sich diese Verhältnisse grundlegend verschoben. Von 37 Briefen reichsdeutscher Absender verwendeten nun 32 den Hitler-Gruß in der Schlussformel. In 27 dieser Fälle steht der Hitler-Gruß am Textende sogar isoliert. Die Schlussformel "Mit deutschem Gruß" finden wir in der Belegsammlung des Jahres 1937 nur noch zweimal, einmal isoliert und einmal in Kombination mit "Heil Hitler". Vier Briefe verwenden weder persönliche Anrede noch Schlussformel. ${ }^{24}$ Die Versicherung der Hochachtung ist aus der Schlussformel der Briefe nun endgültig getilgt. Im Jahr 1937 war das isolierte "Heil Hitler" also zur prototypischen Schlussformel aufgerückt. Es tritt in den hier untersuchten geschäftlichen oder behördlichen Briefen weitgehend unabhängig vom gesellschaftlichen Status des Empfängers auf. In meinen Belegbriefen findet sich der isolierte Hitler-Gruß am Ende von Briefen, mit denen ein "Sehr geehrter Herr Eckert"25 angeschrieben wird, ebenso wie in solchen, die mit der Anrede "Hochverehrter Herr Geheimrat" oder "Spectabilis"26 beginnen. Die knappe Schlussformel "Heil Hitler" ist auch mit einer Vielzahl verschiedener Textfunktionen vereinbar, sie steht am Ende von Bescheiden, Arbeitsberichten und Gutachten ebenso wie von Anfragen, Anträgen und Bitten.

Kombinatorische Erweiterungen des Hitler-Grußes sind im letzten Drittel der 1930er Jahre nur noch vergleichsweise selten. Sie traten beispielsweise auf, wo sich zwischen Fachreferenten der Forschungsgemeinschaft und ihren "Stammkunden" eine persönliche Bekanntschaft entwickelt hatte. So etwa bei Theodor Frings, der Briefe an seinen Ansprechpartner Dr. Horst etwa wie folgt schloss: "Mit freundlichen Empfehlungen / und Heil Hitler / bin ich Ihr [...]"27 Gelegentlich wurde auch der Hitler-Gruß mit Versatzstücken der alten Hochachtungsformel erweitert, wenn ein besonders hoher Status des Briefempfängers markiert werden sollte: So schließen die Bearbeiter des Handwörterbuchs des Grenz- und Auslandsdeutschtums ihren Brief an Ministerialdirektor Vollert vom Innenministerium mit "Heil Hitler! / Ihre sehr erge-

23 Rundschreiben Friedrich Schmidt-Otts an die Ausschüsse der Deutschen Forschungsgemeinschaft vom 14.12.1933 (R73/156: B1. 127).

${ }^{24}$ Die einleitungs- und schlusslosen Schreiben sind typisch für die Korrespondenz zwischen Institutionen, also etwa zwischen Forschungsgemeinschaft und Wissenschaftsministerium, Auswärtigem Amt oder Zollamt.

${ }^{25}$ Bescheid an den Stipendiaten Albert Eckert vom 5.4.1938 (R73/10824).

26 Walther Mitzka an Dekan der Philosophischen Fakultät der Universität Breslau, Meissner, 27.9.1938 (R73/11234).

${ }^{27}$ Brief vom 7.2.1938 (R73/11854), oder 24.5.1937 wieder an Dr. Horst "Heil Hitler! Ihr Sie begrüssender Frings" (R73/10282). 
benen", oder der 80-jährige Edward Schröder grüßt den Präsidenten der Forschungsgemeinschaft, Rudolf Mentzel, mit "Heil Hitler / Hochachtungsvollst"28. In den mit Abstand meisten Fällen verzichten die Briefschreiber meines Belegkorpus aber nach 1937 ganz darauf, den Hitler-Gruß ihrer spezifischen Beziehung zum Briefempfänger anzupassen.

Trotz des massiven politischen Drucks hat die Ablösung der variantenreichen Versicherung der Hochachtung durch das knappe und einförmige "Heil-Hitler" also etwa vier Jahre in Anspruch genommen. Indizien für die Trägheit der ehemaligen Grußkonvention finden wir in zahlreichen Hybridformulierungen, in denen der Ausdruck von Respekt oder Ergebenheit mit der neuen Grußform anfangs häufig noch verbunden wurde. Dabei hat die politisch indifferentere Formel "Mit deutschem Gruß" den Übergang von der Respekterweisung zum Gesinnungsgruß offensichtlich erleichtert. Die Formel "Mit deutschem Gruß" ist in meinem Belegmaterial eindeutig als Übergangslösung zu erkennen, die ab 1934 zunächst öfters allein am Briefsschluss stand, dann zunehmend mit "Heil Hitler" kombiniert wurde, um schließlich ganz hinter den Hitler-Gruß zurück zu treten und selbst außer Gebrauch zu kommen. ${ }^{29}$

Mit der beschriebenen Entwicklung wurde der binnendeutsche Sprachgebrauch nach 1933 von den Verhältnissen im deutschen Sprachgebiet außerhalb der Grenzen des Dritten Reiches abgekoppelt. Ein in Zürich erschienenes Etikette-Buch verfügt 1943 für den distanzierten Abschiedsgruß in Briefen wie gehabt:

Als Schlussformel ist, falls es sich nicht um nähere Bekannte oder Freunde handelt, korrekt:

"Mit vorzüglicher Hochachtung". (Latouche 1943: 51)

Ein deutschstämmiger Gymnasiallehrer aus dem Banat "zeichnet" noch 1939 einen Antrag an die Deutsche Forschungsgemeinschaft "mit dankbarer Hochachtung: / Ihr ergebenster dr. [sic] Ladislaus Weifert"30 und ein sudetendeutscher Stipendiat schließt eine kleine Bitte im selben Jahr mit "Hochachtungsvoll"31. Gerade auch im Bereich der ehemaligen österreichischungarischen Monarchie kommen Briefabsender auch dann nicht ohne die gewohnte Respektbekundung aus, wenn sie im Übrigen Wert auf den Ausdruck ihrer politischen Gesinnung legen: Der Österreicher Rudolph Pettarin, der Mitte der 1930er Jahre "wegen nationalsozialistischer Betätigung"32 aus seiner Anstellung an der Universitätsbibliothek in Wien entlassen worden war, schließt einen Bericht an die Forschungsgemeinschaft 1939 mit den Worten:

Ich bitte die Deutsche Forschungsgemeinschaft die Ausdrücke meiner aufrichtigen Ergebenheit entgegenzunehmen.

Heil dem Führer! ${ }^{33}$

Der sudetendeutsche Nachwuchsromanist Edgar Glässer, der der Linguistik eine Einführung in die rassenkundliche Sprachforschung ${ }^{34}$ bescherte, kombinierte in seinen Briefen noch nach seinem Wechsel von Brünn nach Heidelberg im Jahr 1936 altösterreichische Devotion und die

\footnotetext{
28 Petersen, Ruth und Schwalm am 9.4.1940 an Vollert (R73/11468), und Schreiben Schröders an Mentzel vom 22.8.1939 (R73/10945).

${ }^{29}$ Für behördliche Briefe aus dem Umkreis der Wiener Akademie der Wissenschaften gewann die Formel "Mit deutschem Gruß" nach dem Anschluss Österreichs 1938 schon gar keine Rolle mehr: "Entweder sie verzichteten ganz auf jeden Gruß, was sehr oft vorkam - oder sie endeten mit 'Heil Hitler!'" Auch in der nicht behördlichen Korrespondenz der Akademiemitglieder kam die Formel nach 1938 "nur selten" vor (Graf-Stuhlhofer 1996: 127).

30 Antrag vom 6.5.1939 "An die Deutsche Forschungsgemeinschaft" (R73/ 15561).

${ }^{31}$ Briefkarte, Prag, 14.2.1939 an die Deutsche Forschungsgemeinschaft (R73/11118).

32 Schreiben der Deutschen Gesandtschaft in Wien an die Forschungsgemeinschaft, 15.6.1936 (R73/13584).

33 Dreiseitiger Bericht vom 30.3.1939, ein früherer Brief Pettarins endet am 9.6.1936 "mit dem Ausdruck der ausgezeichneten Hochachtung und dem deutschen Gruße zeichne ich ganz ergebenst" (R73/13584).

34 Verlegt 1939 in Heidelberg durch den Carl Winter Verlag.
} 
Demonstration von Gesinnung gern wie folgt: "Mit den hochachtungsvollst-ergebensten Empfehlungen, / und mit volksdeutschem Gruß, Heil Hitler!"35

\title{
$4 \quad$ Vom Ende der Hochachtung
}

In diesen und ähnlichen hybriden Formulierungen vermischen sich zwei Ausgestaltungen des Briefschlusses, die die Sprachratgeber aus dem Dritten Reich aus prinzipiellen Gründen von einander absetzen wollten. Für sie war der Deutsche Gruß nicht eine lexikalische Zugabe, mit welcher die bisherigen Grußformeln um ein Element von unmittelbar politischer Aussagekraft zeitgemäß erweitert werden konnten. Der Hitler-Gruß wurde vielmehr als radikale historische Überbietung der früheren Grußkonventionen aufgefasst:

\begin{abstract}
Verbräme deine Unterschrift nicht mit gedrechselten und verstiegenen Grußformeln. Früher war man darin peinlich genau [...]. Da "verblieb" man nach strengen Gesetzen "achtungsvoll", "hochachtungsvoll", vielleicht gar "hochachtungsvollst" oder "mit vorzüglicher Hochachtung". [...] Für uns Menschen von heute ein greulicher Zopf, der zu seiner Zeit aber ungeheuer ernst genommen wurde und ein genauer Maßstab war für das Verhältnis zwischen Briefschreiber und Empfänger. Überlassen wir getrost den älteren und ältesten Herrschaften diese feinen Schattierungen.

Mit dem ganzen Plunder hat die neue Zeit aufgeräumt. Was sollen uns heute die verlogenen Beteuerungen von "Achtung", "Hochachtung" und "vorzüglicher Hochachtung". Sie sind und waren immer leer und hohl wie ausgeblasene Eier. Weg damit! Heute sollst du in deinem Brief keine unaufrichtigen Verbeugungen machen. Jeder Deutsche setzt heute unter seinen Brief: "Mit Deutschem Gruß" oder "Heil Hitler". Dieser Gruß gilt jedem ohne Rang- und Standesunterschied. Das ist der Gruß der Volksgemeinschaft [...]. (Elwenspoek 1936: 32-33)
\end{abstract}

Auch Haluschkas Buch für Anständige sieht in der Versicherung der Hochachtung und Ergebenheit am Briefschluss "verstaubte Etikette" und "unterwürfige Formeln, die der heutigen Zeit widersprechen" (Haluschka 1938: 39f.). Ähnlich findet der Verfasser von Die Kunst Briefe zu schreiben, den Ausdruck der "vollsten Ergebenheit" in Bewerbungsbriefen 1939 "einfach lächerlich":

Weiter hinterlässt die kriecherische Unterwürfigkeit im Bewerbungsschreiben einen üblen Eindruck. [...] Bescheidenheit ist etwas sehr schönes und kostbares, Unterwürfigkeit zeugt von wenig Charakter. (Wilda 1939: 88)

Die Kombination "Mit Deutschem Gruß / Ihr ergebenster" taucht in diesem Briefsteller daher nur in einem Negativbeispiel für misslungene Briefgestaltung auf, und wird in dessen vorbildlicher Umformulierung folgerichtig zu einem einfachen "Heil Hitler" (ebd.: 90-91) verkürzt.

Hinter der recht radikal durchgesetzten und ebenso radikal gerechtfertigten Tilgung der hochachtungsvollen und ergebenen Abschiedsgrüße aus dem reichsdeutschen Brief steht die Ablehnung eines Prinzips kommunikativen Umgangs. In den Varianten der Formel "mit vorzüglicher Hochachtung / Ihr ergebener" wurde - durchaus zu Recht - ein Relikt einer Höflichkeit gesehen, die im Wesentlichen auf Strategien der Partnererhöhung und der Selbsterniedrigung basierte und damit vor allem Wert darauf legte, soziale Ungleichheiten in "feinen Schattierungen" zu inszenieren. Noch am Beginn des zwanzigsten Jahrhunderts lässt ein Universalbriefsteller vorbildliche Bittbriefe zwischen Schlussformeln wie den folgenden variieren:

Mich der Hoffnung getröstend, mein ergebenstes Gesuch von Eurer Hochgräflichen Gnaden erfüllt zu sehen,[...] habe ich die Ehre, in tiefster Untertänigkeit zu verharren / Eurer Hochgräflichen Gnaden / ganz gehorsamster Diener

\footnotetext{
35 Brief an die Forschungsgemeinschaft vom 10.2.37, in einem Folgeschreiben wird der Hitler-Gruß zusätzlich gesperrt gedruckt (15.3.37), nahezu wortgleich schon im Schreiben vom 25.6.1936 (R73/11243).
} 
In tiefster Ehrfurcht verharre ich / Euer Hochwohlgeboren / ganz gehorsamster Diener

Geneigter Antwort entgegensehend, verharre ich mit Hochachtung / Euer Wohlgeboren / ergebenster (Wagner 1905: 82, 83, 87).

Die unverkennbar aus der Ständegesellschaft überkommene Höflichkeit kommunizierte die Beziehungen zwischen Gesprächspartnern in Dimensionen einer "Machtsemantik"36 (Brown/Gilman 1982). Mit verbalen, gestischen, proxemischen und sogar typographischen Ausdrucksformen wie Titulatur, Verbeugung, Gewährung des Vortritts oder Großschreibung ehrender Attribute wurden diese Beziehungen als Grade vertikaler gesellschaftlicher Distanz charakterisiert. Bevorzugte Orte entsprechender sozialer Markierung waren gerade auch die Anfangs- und Schlussphasen von Gesprächen und Texten, die typischerweise in besonderem Maße der kommunikativen Beziehungsarbeit gelten. ${ }^{37}$ Die sich kulturrevolutionär gebärdenden Benimm- und Ratgeberbücher der NS-Zeit verwahrten sich grundsätzlich gegen die Prinzipien "dieses klassenmäßigen Anstandes" (Haluschka 1938: 38). Der Deutsche Gruß war also nicht nur deshalb ein politischer Gruß, weil er bei jeder Begegnung das "Gedenken an Führer und Vaterland" (Elwenspoek 1936: 113) in Worte und Gesten fasste, sondern weil er in seiner gewollten Respektlosigkeit und Gleichförmigkeit der egalitären Utopie der Volksgemeinschaft entsprach.

Nun ist aber die Polemik gegen "speichelleckerische Redensarten" (Elwenspoek 1936: 44) der überkommenen Höflichkeit keineswegs erst im Kontext des Nationalsozialismus aufgekommen. ${ }^{38}$ Vielmehr durchziehen mitunter vehemente Ausfälle gegen die "Kriecherei" der ständischen Höflichkeit den Anstandsdiskurs schon seit dem späten 18. Jahrhundert. ${ }^{39}$ Immer wieder wird in der deutschen Ratgeberliteratur des 19. und frühen 20. Jahrhunderts davor gewarnt, "heuchlerisch, kriechend oder auch nur auf unerlaubte Art schmeichlerisch" (Falckmann 1838: 245) vorzugehen, und es wird dringend empfohlen, man sei "im Gebrauche aller Höflichkeits- und Ehrerbietungszeichen verständig" (Wagner 1875: 328). Die "zu kriechenden und sklavischen Äußerungen und Höflichkeiten" würden "nur einen unangenehmen Eindruck" (Kiesewetter 1865: 58) oder "sogar das Gefühl des Widerwillens" (Wilm et al. 1927: $3)^{40}$ hervorrufen. Im Hintergrund dieses stark emotional besetzten Kriecherei-Verbotes steht mehr oder weniger explizit ein bürgerlicher Grundwert:

Die allzu große Demüthigung und Erniedrigung verletzt die eigene Würde des Menschen. (Wagner 1875: 328)

Auch wenn die bestehenden Verhältnisse den Menschen noch lange Zeit das kommunikative Kunststück abverlangten, "sich gebührend unter[zu]ordnen und [zu] unterwerfen, ohne einen

\footnotetext{
36 Roger Brown und Albert Gilman (1982) zeigen in ihrer klassischen Studie zu den Anredepronomen in verschiedenen europäischen Sprachen den gesellschaftsgeschichtlich motivierten Übergang von der "Machtsemantik" zur "Solidaritätssemantik". "Eine starke Gleichheitsideologie [...] führt dazu, jeden herkömmlichen Ausdruck von Machtasymmetrie zu unterdrücken" (ebd.: 185).

37 Vgl. Laver (1981: 296): "In the marginal phases [of conversation], where greeting and parting are negotiated, social factors will loom largest, with propositional mechanisms beeing subordinated to a social purpose." Zur räumlichen und graphischen Gestaltung von Briefen im 19. und 20 Jahrhundert vgl. Ehlers (2004).

38 Hier ist Brüggemann (1968: 27) zu widersprechen, der behauptet: "Erst die Briefsteller zwischen 1933 und 1945 wollten unter Hinweis auf die Volksgemeinschaft die Duckmäuserei austreiben".

${ }^{39}$ In der Brieflehre ist der Beginn der Kriecherei-Kritik natürlich in den wirkungsmächtigen Texten Gellerts zu verorten, der unter anderem auch gegen die untertänigen Schlussformeln argumentiert, vgl. Haß-Zumkehr (1999: 260-261).

40 Nicht nur die Benimmbücher sprechen davon, dass die Kriecherei "in den allermeisten Fällen einen abstoßenden Eindruck" hervorrufe, Otto (o.J.: 49). Auch Georg Steinhausens am Ende des 19. Jahrhunderts erschienene Geschichte des deutschen Briefes ist über weite Passagen eine rabiate Polemik gegen die "widerwärtige und geschmacklose Komplementiererei" früherer Zeiten und die "'Hundedemut', die leider eine Zeitlang als Charakterzug der Deutschen gegolten hat" (Steinhausen 1891: 52). Die Kritik der ständischen Höflichkeitsformen hat bei Steinhausen eine starke antifranzösische Tendenz.
} 
erniedrigenden und entehrenden knechtischen Sinn zu zeigen" (ebd.), war der Anstandsdiskurs im 19. Jahrhundert doch schon weitgehend auf eine bürgerliche Wertordnung perspektiviert. Während das ständische Kommunikationsverhalten "vor allem die gesellschaftliche Ungleichheit" (Linke 1998: 143) zum Orientierungspunkt hatte, entwickelte das aufkommende Bürgertum eine "Höflichkeit unter Gleichgestellten", in deren Zentrum das "Prinzip der Diskretion und Distanz" (ebd.: 144) stand. Auch die selbstverständlich weiterhin bestehenden Statusunterschiede wurden nun zunehmend als Grade der horizontalen gesellschaftlichen Distanz kommuniziert. Auf der Ebene der formalen Mittel war der Übergang von der ständischen Höflichkeit der Ungleichheit zur bürgerlichen Höflichkeit der Gleichheit durch eine Reduktion der Variationsmöglichkeiten und eine Verringerung des Aufwands von Respekterweisungen gekennzeichnet. Diese Entwicklung verlief für verschiedene höfliche Routinen zum Teil phasenverschoben und reichte mitunter noch weit in das zwanzigste Jahrhundert hinein. ${ }^{41}$ So ist die um 1930 noch gebräuchliche Schlussformel "mit vorzüglicher Hochachtung Ihr ergebener" bereits eine späte Schwundstufe viel ausladend ausgeführter Vorläufer. Wenn die Sprachlenkung des Nationalsozialismus diese ausgedünnte Reduktionsform endgültig durch den Deutschen Gruß verdrängte, dann beschleunigte bzw. vollendete sie eine Entwicklung des Sprachgebrauchs, die sich schon mehr als ein Jahrhundert früher abzuzeichnen begonnen hatte. Paradoxerweise brachte so ein politisches System, das sich herausnahm, Bürgerrechte und Menschenwürde exklusiv zu vergeben, eine zutiefst bürgerlich geprägte Entwicklung der Kommunikationsformen zu ihrem Abschluss. Wie in anderen gesellschaftlichen und wirtschaftlichen Bereichen zeigte der Nationalsozialismus auch im Feld der kommunikativen Routinen seine eigentümliche Verbindung von Regression und Modernisierung. ${ }^{42}$

Nach 1945 beeilten sich deutsche Benimmbücher und Briefsteller allerdings zunächst, die Grußkonventionen der Zeit vor 1933 wieder in Kraft zu setzen. Vom Deutschen Gruß ist nun nicht einmal mehr im negativen Sinne noch zu lesen. Schon in der ausufernden Ratgeberliteratur der 1950er Jahre erschien die Versicherung der Hochachtung am Schluss geschäftlicher Briefe aber gelegentlich als "starr und farblos" (Chamrath 1954: 55) und eine weitere Reduktion auf ein einfaches "Hochachtungsvoll" bahnte sich bald an. Briefsteller der 1970er und frühen 1980er Jahre sahen in den Rückzugsdomänen der Hochachtung und Ergebenheit dann wieder "Überbleibsel einer vergangenen Zeit" (Wachtel o.J.: 79) und "alte Briefzöpfe", die endlich "abgeschnitten gehörten" (Umgangsformen 1982), weil "die Zeiten der Leibeigenschaft, der Frondienste und des Gesindezwangsdienstes [...] lange vorbei" (Graudenz 1971: 30) seien. Wenn die Versicherung der Hochachtung bis heute fast vollständig aus dem bundesdeutschen Sprachgebrauch verschwunden ist, ${ }^{43}$ dann vollendete die mehr oder weniger ungesteuerte Sprachentwicklung der Nachkriegszeit, was der Nationalsozialismus vorzeitig mit Gewalt durchzusetzen versucht hatte: die endgültige Herauslösung des Briefschlusses aus der Entwicklungsgeschichte der ständischen Höflichkeit.

Anders als der idealerweise isolierte und einförmige Hitler-Gruß ermöglichen die neuen Formen "Mit freundlichen Grüßen" "Mit herzlichen Grüßen" "Mit liebem Gruß" usw. aber wie-

\footnotetext{
${ }^{41}$ In ihrem Buch über die Sprachkultur des Bürgertums beobachtet Angelika Linke die Entwicklung der "bürgerlichen Höflichkeit" u.a. an dem allmählichen Übergang "vom Compliment zum Gruss", bei dem die Reduktion der Respekterweisung zugleich mit einer Verlagerung von gestischen auf sprachliche Ausdrucksmittel einhergeht (Linke 1996: 104-131). Für den Bereich des deutschen Anredeverhaltens beschreibt Besch (2003) die Ablösung der "Hofschranzen-Höflichkeit". Einer parallelen Entwicklung unterliegt der Wandel der räumlichen Gestaltungsmittel in Briefen, der ebenfalls auf eine Reduktion der Respektsemiotik hinauslief, vgl. Ehlers (2004).

42 Zur "Ambivalenz der Moderne im Nationalsozialismus" vgl. den Forschungsbericht von Bavaj (2003).

43 Von 190 Antwortschreiben auf eine Wohnungsannonce, die Reindl im Jahr 1994 untersuchte, wählte nur ein einziger Schreiber als Schlussformel seines Bewerbungsschreibens "Hochachtungsvoll". Als "Korpusnorm" galt vielmehr der Abschlussgruß "Mit freundlichen Grüßen" (Reindl 1994: 174).
} 
der eine feine Differenzierung der sozialen Beziehungen - nun aber endgültig in Dimensionen einer horizontalen Distanz.

\section{Literatur}

Allert, Tilman (2005): Der deutsche Gruß. Geschichte einer unheilvollen Geste. Berlin: Eichborn.

Bauer, Gerhard (1988): Sprache und Sprachlosigkeit im "Dritten Reich". Köln: Bund Verlag.

Bavaj, Riccardo (2003): Die Ambivalenz der Moderne im Nationalsozialismus. Eine Bilanz der Forschung. Mit einem Nachwort von Klaus Hildebrand. München: Oldenbourg.

Besch, Werner (2003): "Anredeformen des Deutschen im geschichtlichen Wandel." In: Sprachgeschichte. Ein Handbuch zur Geschichte der deutschen Sprache und ihrer Erforschung. 2. Aufl. hrsgg. von Werner Besch et al. Berlin, New York: De Gruyter: 25992628.

Briefe, die Eindruck machen. Anleitung zur Abfassung wirkungsvoller und erfolgversprechender Briefe. Privatbriefe von Carl Freymann. Geschäftsbriefe und Bewerbungsschreiben von Gustav Lehmann. Schreiben an Behörden, Eingaben und Urkunden von Fritz Manke. Dresden, 1936: Rudolph'sche Verlagsbuchhandlung.

Brown, Roger/Gilman, Albert (1982): "Die Pronomina der 'Macht' und der 'Solidarität'." Deutsch in: Steger, Hugo (Hrsg.): Anwendungsbereiche der Soziolinguistik. Darmstadt: Wissenschaftliche Buchgesellschaft: 163-198.

Brüggemann, Diethelm (1968): Vom Herzen direkt in die Feder. Die Deutschen in ihren Briefstellern. München: dtv.

Chamrath, Gustav (1954): Lexikon des guten Tones. 2. Aufl. Wien: Ullstein.

Ehlers, Klaas-Hinrich (2004): "Raumverhalten auf dem Papier. Der Untergang eines komplexen Zeichensystems dargestellt an Briefstellern des 19. und des 20. Jahrhunderts." In: Zeitschrift für germanistische Linguistik 32 (2004): 1-31.

Eibl-Eibesfeldt, Irenäus (1971): "Zur Ethologie menschlichen Grußverhaltens: II. Das Grußverhalten und einige andere Muster freundlicher Kontaktaufnahme der Waika (Yanoáma)." In: Zeitschrift für Tierpsychologie 29: 196-213.

Elwenspoek, Curt (1936): Der rechte Brief zur rechten Zeit. Eine Fibel des schriftlichen Verkehrs für jedermann. Leipzig: Hesse \& Becker.

Ermert, Karl (1979): Briefsorten. Untersuchungen zu Theorie und Empirie der Textklassifikation. Tübingen: Niemeyer.

Falckmann, Ch. F. (1838): Stylistisches Elementarbuch oder: Erster Cursus der Stylübungen, enthaltend: eine kurze Anleitung zum guten Styl, eine große Anzahl Aufgaben sowohl zu einzelnen Vorübungen, als auch zu Beschreibungen, Erzählungen, Abhandlungen, Briefen und Geschäfftsaufsätzen aller Art [...], für Anfänger im schriftlichen Vortrage und zur Selbstbelehrung bestimmt. 5. Aufl. Hannover: Hahnsche Hofbuchhandlung.

Freymann, Carl (1936): "Privatbriefe. Anleitung zur Abfassung wirkungsvoller Privatbriefe für alle Gelegenheiten." In: Briefe, die Eindruck machen. Dresden: Rudolph'sche Verlagsbuchhandlung: 5-128.

Graf-Stuhlhofer, Franz (1996): "'Heil Hitler!' am Schluß von Briefen." In: Österreich in Geschichte und Literatur 40: 125-128.

Graudenz, Karlheinz (1971): Die Briefetikette. München: Südwest Verlag.

Grosse, Siegried u.a. (Hrsg.): "Denn das Schreiben gehört nicht zu meiner täglichen Beschäftigung". Der Alltag kleiner Leute in Bittschriften, Briefen und Berichten aus dem 19. Jahrhundert. Ein Lesebuch. Bonn: Dietz.

Haluschka, Helene (1938): Noch guter Ton? Ein Buch für Anständige. Graz: Ulrich Moser.

Haß-Zumkehr, Ulrike (1999): "Die kulturelle Dimension der Lexikografie. Am Beispiel der Wörterbücher von Adelung und Campe." In: Gardt, Andreas/Haß-Zumkehr, Ulrike/Roelcke, Thorsten (Hrsg.): Sprachgeschichte als Kulturgeschichte. Berlin, New York: De Gruyter: 247-265. 
Kiesewetter, L. (1865): Neuer praktischer Universal-Briefsteller für das geschäftliche und gesellige Leben. Ein Formular- und Musterbuch zur Abfassung aller Gattungen von Briefen, Eingaben, Contracten [...] Mit genauen Regeln über Briefstyl überhaupt und jede einzelne Briefgattung [...].17. Aufl. Glogau: Carl Fleming.

Klemperer, Victor (1957): LTI. Notizbuch eines Philologen. 3. Aufl. Halle (Saale): Niemeyer.

Klemperer, Victor (1995): Ich will Zeugnis ablegen bis zum letzten. Tagebücher 1933-1941. Hrsgg. von Walter Nowojski unter Mitarbeit von Hadwig Klemperer. 2. Aufl. Berlin: Aufbau.

Krumrey, Horst-Volker (1984): Entwicklungsstrukturen von Verhaltensstandarden. Eine soziologische Prozessanalyse auf der Grundlage deutscher Anstands- und Manierenbücher von 1870 bis 1970. Frankfurt am Main: Suhrkamp.

Latouche, Annette (1943): Etikette. Von geistigem und gesellschaftlichem Takt. Zürich: Verlag Amstutz Herdig.

Laver, John (1981): "Linguistic Routines and Politeness in Greeting and Parting." In: Coulmas, Florian (Hrsg.): Conversational Routine. Explorations in Standardized Communication Situations and Prepatterned Speech. The Hague, Paris, New York: Mouton: 289-304.

Leder, Heinz (o.J.): Du und Deine Volksgenossen. Ein Wegweiser zu neuzeitlichen Umgangsformen. Minden, Westfalen: Köhler.

Lehmann, Gustav (1936): "Geschäftsbriefe. Anleitung zur Abfassung guter Geschäftsbriefe für Handel- und Gewerbetreibende." In: Briefe, die Eindruck machen. Dresden: Rudolph'sche Verlagsbuchhandlung: 129-239.

Linke, Angelika (1996): Sprachkultur und Bürgertum. Zur Mentalitätsgeschichte des 19. Jahrhunderts. Stuttgart, Weimar: Metzler.

Linke, Angelika (1998): "Sprache, Gesellschaft und Geschichte. Überlegungen zur symbolischen Funktion kommunikativer Praktiken der Distanz." In: Zeitschrift für germanistische Linguistik 26 (1998): 135-154.

Mancke, Fritz (1936): "Schreiben an Behörden, Eingaben und Urkunden. Anleitung und Ratgeber für den Schriftverkehr mit Behörden." In: Briefe, die Eindruck machen. Dresden: Rudolph'sche Verlagsbuchhandlung: 241-329.

Martin, Hans (o.J. [NS-Zeit]): Darf ich mir erlauben...? Das Buch der guten Lebensart. Mit vielen Bildern und praktischen Beispielen. Stuttgart: Süddeutsches Verlagshaus.

Meister, Ilse (1934 [?]): Der praktische Schreibtischberater. Ein Nachschlagebuch zum täglichen Gebrauch für jedermann. Stuttgart, Berlin, Leipzig: Union Deutsche Verlagsgesellschaft.

Overesch, Manfred et al. (2001): Das Dritte Reich. Daten - Bilder - Dokumente. Eine Tageschronik mit 1.700 Abbildungen aus dem Bildarchiv Heinz Bergschicker. Von Manfred Overesch, Friedrich Wilhelm Saal, Wolfgang Schneider und Bernd Weinkauf. Berlin: directmedia. (=Digitale Bibliothek Bd. 49).

Reindl, Christine (1994): "'Über eine Antwort würde ich mich sehr freuen.' Untersuchung von Antworten auf ein Chiffre-Wohnungsangebot." In: Lütten-Gödecke, Jutta/Zillig, Werner (Hrsg.): "Mit freundlichen Grüßen". Linguistische Untersuchungen zu Problemen des Briefe-Schreibens. Münster: AA-Verlag: 111-192.

Rohr, J. (o.J. [nach 1938]): Rede, schreibe, rechne richtig! Mit einem deutschen Sprachschatz von etwa 60000 Wörtern. Berlin: Deutsche Buch-Gemeinschaft.

Scharloth, Joachim/Okamura, Saburo (2010): "Die feinen Unterschiede. Zur sozialen Konstruktivität von Begrüßungsritualen im Japanischen unter formal Statusgleichen." In: Zeitschrift für angewandte Linguistik 52 (2010): 49-79.

Steinhausen, Georg (1891): Geschichte des deutschen Briefes. Zur Kulturgeschichte des deutschen Volkes. Zweiter Teil. Unveränderter Nachdruck. Dublin, Zürich 1968: Weidmann.

Teichmann, Hans (o.J. [NS-Zeit]): Der wirklich brauchbare Ratgeber für richtiges Benehmen und gute Umgangsformen. Dresden: Rudolph'sche Verlagsbuchhandlung. 
Umgangsformen heute. Die Empfehlungen des Fachausschusses für Umgangsformen. Überarb. u. erg. Neuaufl. Niedernhausen 1982: Falken.

Wachtel, Joachim (o.J. [nach 1974]): 1 x 1 des guten Tons heute. München, Gütersloh, Wien: Bertelsmann.

Wagner, Ernst (1905): Neuer praktischer Briefsteller oder Ratgeber und Musterbuch zur richtigen Abfassung aller im bürgerlichen Leben und im Geschäft vorkommenden Briefe, Eingaben, Verträge, Urkunden und anderer Aufsätze. Zugleich Ratgeber in Rechtsangelegenheiten [...]. Stuttgart: Fleischhauer \& Spohn.

Wagner, Fridolin (1875): Die Lehre vom deutschen Stil oder praktische Anleitung zum richtigen deutschen Gedankenausdrucke für die oberen Classen der Volksschulen, höheren Mädchenschulen und einzelnen Classen der Realanstalten und Gymnasien, wie zum Privatgebrauche. 10. Aufl. Darmstadt: Johann Philipp Diehl.

Wilda, Harald van (1939): Die Kunst Briefe zu schreiben. Neubearbeitet von Dr. Hanns Martin Elster. Dresden: Rudolph'sche Verlagsbuchhandlung.

Wilm, Werner u.a. (1927): "Der geschäftliche, öffentliche und private Schriftwechsel. Mit praktischen Übungen." In: Rede und Schrift. Handbuch und Nachschlagewerk für die allgemeinen Wissensgebiete des öffentlichen und des privaten Lebens. Bd. 2. Leipzig: Weiman: 1-296. 